\title{
Bail-out use of the Amplatzer Septal Occluder for treatment of acute iatrogenic leaflet perforation during the MitraClip procedure in a patient with functional mitral regurgitation
}

\author{
Adrian Kłapyta ${ }^{1}$, Jerzy Pręgowski², Zbigniew Chmielak², Piotr Szymański ${ }^{3}$, Adam Witkowski², Marcin Demkow \\ ${ }^{1}$ Freelance \\ ${ }^{2}$ Department of Interventional Cardiology and Angiology, Institute of Cardiology, Warsaw, Poland \\ ${ }^{3}$ Department of Acquired Heart Diseases, Institute of Cardiology, Warsaw, Poland \\ ${ }^{4}$ Department of Coronary and Structural Heart Diseases, Institute of Cardiology, Warsaw, Poland
}

Adv Interv Cardiol 2018; 14, 3 (53): 304-308

DOI: https://doi.org/10.5114/aic.2018.78336

\section{Introduction}

Surgery is the treatment of choice for patients with significant symptomatic mitral regurgitation (MR). However, up to $50 \%$ of them are denied the procedure due to high operative risk [1]. The MitraClip procedure has been recently widely adopted for the treatment of symptomatic patients with significant degenerative and functional mitral regurgitation who are deemed inoperable [2]. Percutaneous edge-to-edge repair is an efficient technique for the majority of patients with significant MR. Nevertheless, there are still cases with residual significant mitral regurgitation after the device deployment, which cannot be fixed with additional clip implantation (i.e. significant jets in the commissural areas). Recently, a novel procedure was reported using the Amplatzer Duct Occluder II or Amplatzer Vascular Plug for residual MR after MitraClip implantation $[3,4]$. Kubo et al. presented 9 patients treated electively for residual jets localized either between previously implanted clips or in the commissural areas [3].

In the main, the MitraClip procedure is safe with a 30day mortality rate up to $3 \%$ and a relatively small number of serious complications. Even so, in some cases it may occur and the operators should be able to fix them. The treatment of acute iatrogenic leaflet perforation with the implantation of an Amplatzer septal occluder (ASO) during a complicated MitraClip procedure is presented in the current report.

\section{Case report}

The 74-year old woman was referred to hospital due to recurrent symptoms of heart failure which twice caused hospitalization during the previous 12 months. The patient had a history of anterior myocardial infarction that was treated with primary $\mathrm{PCI} 7$ years ago. Other comorbidities included diabetes mellitus, chronic kidney disease grade III and a history of colorectal cancer successfully treated 3 years ago. Recent coronary angiography did not reveal obstructive disease that required revascularization. The transthoracic echocardiography (TTE) showed left ventricular enlargement (left ventricular end diastolic diameter $=61 \mathrm{~mm}$ ), depressed systolic function ( $E F=25 \%$ ), left atrial enlargement, severe functional mitral regurgitation, moderate tricuspid regurgitation and right ventricular systolic pressure of $70 \mathrm{~mm} \mathrm{Hg}$. The therapy for a heart failure in accordance with the guidelines was prescribed to the patient and consisted of full doses of $\beta$-blockers, angiotensin-converting enzyme inhibitor (ACE) and eplerenone. A cardioverter-defibrillator was implanted in the patient for the primary prevention of sudden cardiac death. There was no indication for resynchronization therapy. Due to the fact that heart failure symptoms were persistent, the decision was made to treat mitral regurgitation interventionally. Regards the operation risk, the calculated STS score for mortality was $6 \%$. Following the Heart Team discussion and considering the patient's preferences, MitraClip device implantation was decided. The pre-procedural transesophageal echocardiography (TEE) confirmed significant mitral regurgitation and the mitral valve anatomy was suitable for the MitraClip therapy (Figure 1 A).

The MitraClip procedure was performed under general anesthesia. In addition fluoroscopy and TEE were used

Corresponding author:

Adrian Kłapyta, 21 B Postępu St, 02-676 Warsaw, Poland, phone: +48 500738 644, e-mail: adrian.a.klapyta@gmail.com

Received: 18.10.2017, accepted: 11.04.2018. 

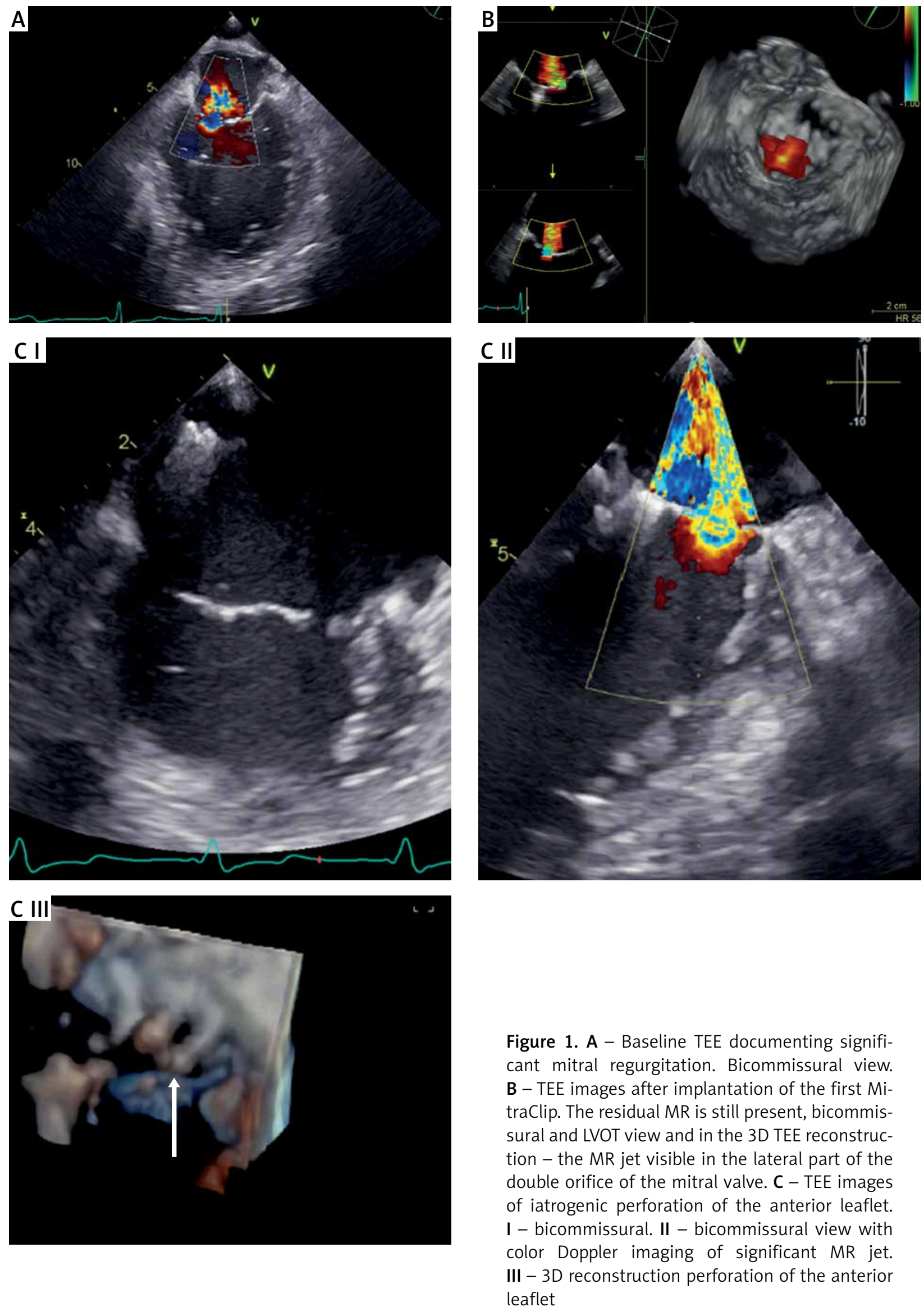

Figure 1. A - Baseline TEE documenting significant mitral regurgitation. Bicommissural view. B - TEE images after implantation of the first MitraClip. The residual MR is still present, bicommissural and LVOT view and in the 3D TEE reconstruction - the MR jet visible in the lateral part of the double orifice of the mitral valve. $\mathbf{C}$ - TEE images of iatrogenic perforation of the anterior leaflet. I - bicommissural. II - bicommissural view with color Doppler imaging of significant MR jet. III - 3D reconstruction perforation of the anterior leaflet 
to guide the procedure. The first MitraClip was implanted into the central aspect of the mitral valve (A2-P2 area). There was substantial residual MR following the implantation of the first clip (Figure $1 \mathrm{~B}$ ) and therefore the decision was made to implant the second clip laterally. After positioning to achieve perpendicularity and the proper axial pathway, clip arms were closed and the device was advanced into the left ventricle. Afterwards, the clip was re-opened and slowly upraised towards the leaflets. Nevertheless, unexpected resistance occurred due to the clip's entanglement within the tendinous chords. The clip was opened to the inverted position in order to remove it safely into the left atrium. However, the subsequent attempts to retract the clip continued to fail despite the various maneuvers. Finally, the clip was retracted into the left atrium, but, unfortunately, the TEE revealed a new large regurgitation jet which originated from

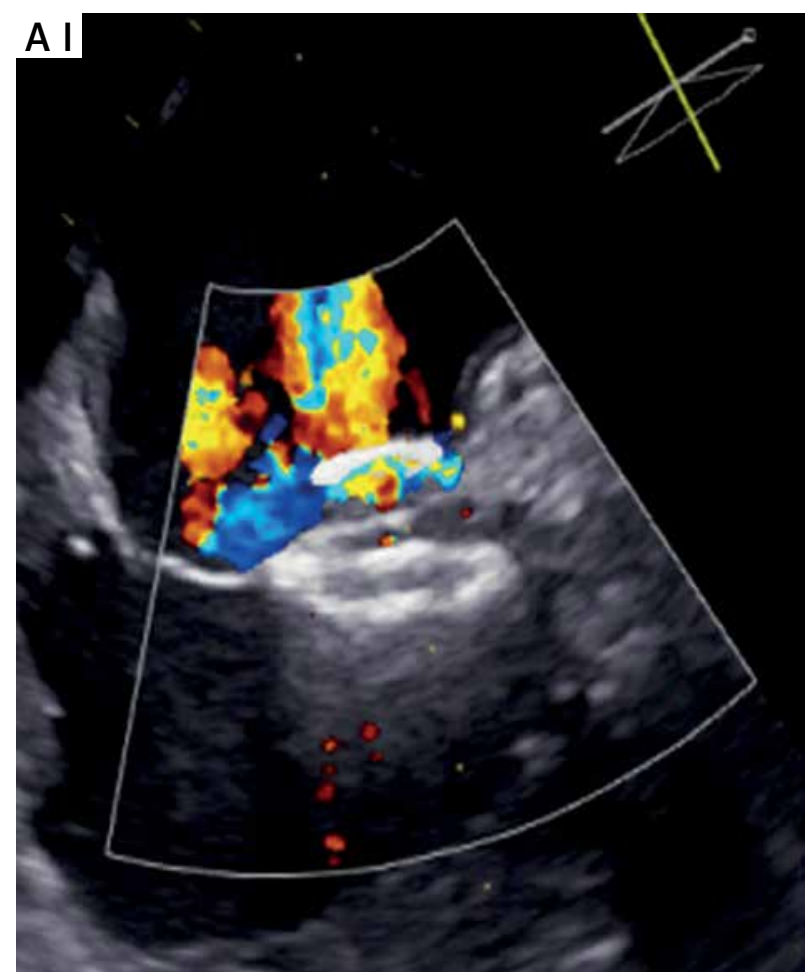

B

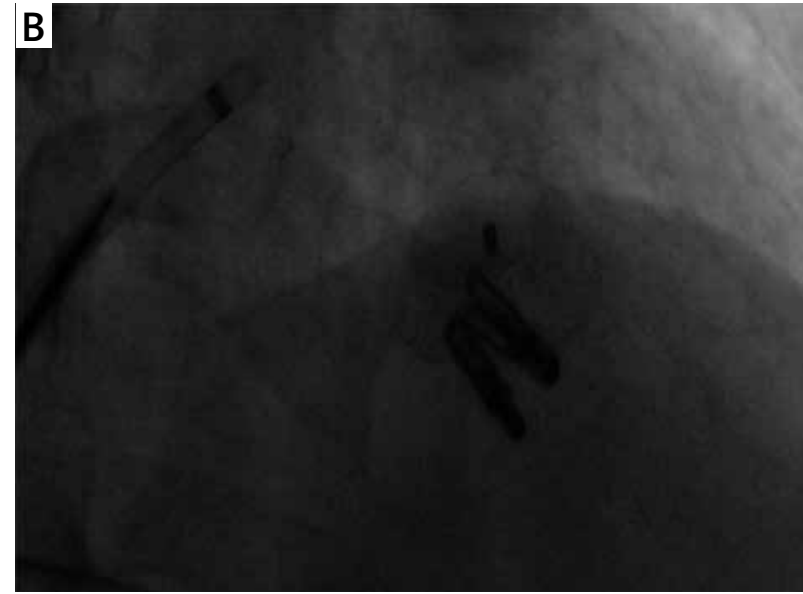

the perforation of the anterior leaflet (Figure $1 \mathrm{C} \mathrm{I-III).}$ To assess and size the perforation, 3D TEE with volume rendering was used. The patient's hemodynamics deteriorated and i.v. inotropic support with dobutamine infusion was required. Following the discussion with cardiac surgeons, the decision was made to continue with a percutaneous procedure in view of the extremely high risk of emergency surgery. The bail-out plan was implemented and the second clip was placed laterally, next to the first one, in order to stabilize the position of both devices. The implantation of the second clip was successful without additional complications. Therefore, after two-dimensional and three-dimensional echocardiographic measurements the decision was made to use the $11 \mathrm{~mm}$ ASO device to cover residual mitral regurgitation caused by the perforation of the anterior leaflet. Using the MitraClip delivery sheath, the catheter and the guidewire

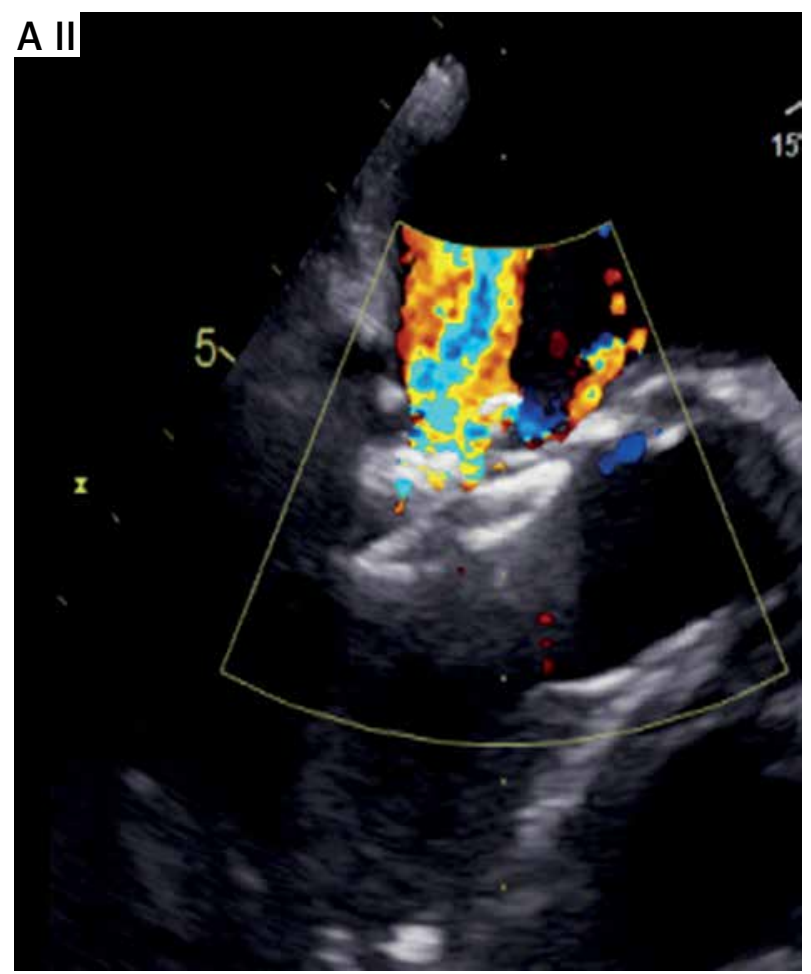

Figure 2. A - TEE images after implantation of the Amplatzer septal occluder (11 mm) into the lateral orifice of the mitral valve. I- bicommissural view, II - LVOT view. B - Angiographic image of the final effect of the procedure: two MitraClips and ASO device implanted laterally 
were inserted into the left ventricle through the lateral orifice of the mitral valve. Over the guidewire the ASO device was delivered into the left ventricle, positioned between the lateral clip and the lateral commissure, and deployed appropriately. A few attempts to grasp the anterior leaflet with the perforation between the discs of the ASO device were performed; nonetheless, its position was suboptimal. The part of the posterior leaflet was not captured between the retention discs (Figure 2 A I-II). A new coaptation line was created between the posterior leaflet and the ASO device. Overall, the grade of mitral regurgitation was substantially reduced without causing a significant transvalvular gradient. The trans-mitral gradient immediately following completion of the procedure was $6.3 / 3.5 \mathrm{~mm} \mathrm{Hg}$ in TEE measurement. As the hemodynamic parameters were stabilized, the patient was extubated. However, during the first hours after the procedure severe hemolysis developed and required massive blood transfusions to the patient. The transthoracic echo showed a stable position of both clips and ASO with the coexistence of moderate mitral regurgitation and a trans-mitral gradient of 8.2/2.6 $\mathrm{mm} \mathrm{Hg}$. Despite the treatment with intravenous fluids and bicarbonates, on the second day continuous venovenous hemofiltration was necessary as the subject developed acute renal failure with anuria as a complication of severe hemolysis (Table I). Following an additional Heart Team meeting the patient was qualified for urgent high-risk mitral valve replacement. The procedure was performed with implantation of a valve bioprosthesis (Hancock II $29 \mathrm{~mm}$, Medtronic). After the surgery the patient was extubated but required extracorporeal membrane oxygenation support for the hemodynamic instability that was unresponsive to the high doses of ionotropic drugs. The renal replacement therapy was continued. Despite the intensive therapy the signs of multi-organ failure were worsening and on the ninth day after surgery the patient died.

\section{Discussion}

To the best of our knowledge, this is the first report of bail-out percutaneous treatment of iatrogenic leaflet perforation complicating MitraClip implantation. The mechanism of the complication is not fully understood. The implanting team speculated that during attempts of retrieval of the entangled device from the left ventricle the forces applied to the chords were transmitted to the leaflet and led to its perforation. The idea to treat the complication with the occluder device was based on the reports of the successful treatment of residual intra-clip and peri-commissural mitral regurgitation with the Amplatzer Vascular Plug II (AVP II) and Amplatzer Duct Occluder II (ADO II) [3, 4]. Kubo et al. presented a series of 9 consecutive patients successfully treated with ADO II for residual MR. The size of the device used ranged from $5 / 4$ to $6 / 6 \mathrm{~mm}$. The implanters hoped that the rel-
Table I. Biochemical results

\begin{tabular}{lcccc} 
Parameter & Baseline & Day 1 & Day 2 & Day 3 \\
\hline Hemoglobin $[\mathrm{g} / \mathrm{dl}]$ & 10.5 & 7.9 & 6.7 & 9.2 \\
\hline Creatinine $[\mu \mathrm{mol} / \mathrm{l}]$ & 101 & 218 & 256 & 233 \\
\hline Total bilirubin $[\mu \mathrm{mol} / \mathrm{l}]$ & 16.8 & 131.2 & 285.3 & 387.3 \\
\hline Free bilirubin $[\mu \mathrm{mol} / \mathrm{l}]$ & & 116.3 & & \\
\hline Conjugated bilirubin & & 12.7 & &
\end{tabular}

atively large retention discs of the $11 \mathrm{~mm}$ ASO would cover the ruptured part of the leaflet and fix the large iatrogenic MR. The acute result of the bail-out procedure was promising as the mitral regurgitation diminished to a moderate grade and the patient's hemodynamic status improved, too. However, the further course was complicated by permanent massive intravascular hemolysis that led to acute renal failure and eventually the decision of rescue surgery (Table I).

Intravascular hemolysis is a common complication following placement of intracardiac devices. It was described after mechanical valve implantation, surgical and percutaneous treatment of ventricular septal defect and patent arterial ducts [4-8]. The turbulent flow through the device and associated high shear stress cause damage to blood cells that release free hemoglobin, which in turn impairs the function of nephrons. In the majority of cases reported the renal failure associated with hemolysis is a transient phenomenon that responds to conservative treatment with adequate hydration. Unfortunately, in this case the hemolysis continued and an attempt of rescue high-risk life-saving surgery was required. Thicker struts of the ASO device as compared to ADO and its suboptimal position at the anterior leaflet only are possible explanations for the huge hemolysis that developed.

\section{Conflict of interest}

Adrian Kłapyta is the MitraClip Therapy Specialist, Abbott Laboratories. Other authors declare no conflict of interest.

\section{References}

1. Mirabel M, lung B, Baron G, et al. What are the characteristics of patients with severe, symptomatic, mitral regurgitation who are denied surgery? Eur Heart J 2007; 28: 1358-65.

2. Nickenig G, Estevez-Loureiro R, Franzen O, et al.; Transcatheter Valve Treatment Sentinel Registry Investigators of the EURObservational Research Programme of the European Society of Cardiology. Percutaneous mitral valve edge-to-edge repair: in-hospital results and 1-year follow-up of 628 patients of the 2011-2012 Pilot European Sentinel Registry. J Am Coll Cardiol 2014; 64: 875-84.

3. Kubo S, Cox JM, Mizutani Y, et al. Transcatheter procedure for residual mitral regurgitation after MitraClip implantation us- 
ing Amplatzer Duct Occluder II. JACC Cardiovasc Interv 2016; 9: 1280-8.

4. Taramasso M, Zuber M, Gruner C, et al. First-in-man report of residual "intra-clip" regurgitation between two MitraClips treated by Amplatzer Vascular Plug II. Eurolntervention 2016; 11: 1537-40.

5. Ellis JT, Wick TM, Yoganathan AP. Prosthesis-induced hemolysis: mechanisms and quantification of shear stress. J Heart Valve Dis 1998; 7: 376-86.

6. Ali Khan MA, al Yousef S, Mullins CE, Sawyer W. Experience with 205 procedures of transcatheter closure of ductus arteriosus in 182 patients, with special reference to residual shunts and longterm follow-up. J Thorac Cardiovasc Surg 1992; 104: 1721-7.

7. Martinez MW, Mookadam M, Mookadam F. A case of hemolysis after percutaneous ventricular septal defect closure with a device. J Invasive Cardiol 2007; 19: E192-4.

8. Anil SR, Sivakumar K, Philip AK, et al. Clinical course and management strategies for hemolysis after transcatheter closure of patent arterial ducts. Catheter Cardiovasc Interv 2003; 59: 538-43. 